\title{
Thrombolysis in acute ischemic stroke: Experience from a tertiary care centre in India
}

\author{
Vikram Huded, Romnesh De Souza, Rajesh Karalumangala Nagarajaiah, Syed Moeed Zafer, Rithesh Nair, Hariram Acharya \\ Department of Neurology, Narayana Hrudayalaya Institute of Neurosciences, Bangalore, Karnataka, India
}

\begin{abstract}
The management of acute ischemic stroke has undergone a sea of change with the introduction of intravenous thrombolysis (IVT). Current guidelines state that the window period for IVT using rTPA is 4.5 hours. The MERCI, Multi Merci, and Penumbra trials in which patients with acute ischemic stroke were treated using endovascular treatment demonstrated better recanalisation in patients having a large vessel occlusion. However, recently published data from the three large trials IMS 3 , Synthesis Expansion, and MR rescue, which compared endovascular treatment with intravenous therapy, failed to demonstrate superiority of endovascular treatment over IVT. In these trials, stent retrievers were used in very few patients. We present our results from a tertiary care center in India where patients are treated using intravenous as well as endovascular modalities. Among the 53 patients with acute ischemic stroke treated between 2010 and 2012, 23 were treated with IVT and 30 with endovascular methods. Stent retriever was used in majority of the endovascular cases. Aims: To compare the outcomes of acute ischemic stroke patients treated with IVT versus those who were managed using endovascular therapy. To evaluate outcomes of patients with acute ischemic stroke with a large vessel occlusion in whom endovascular modalities were used and to compare them with those of patients who were treated with IVT in presence of a large vessel occlusion. Settings and Design: Data of patients who underwent thrombolysis at our centre was collected over a 3-year period, that is, from 2010 to 2012. Endovascular treatment was done by an interventional neurologist. Materials and Methods: Data of patients with acute ischemic stroke who underwent IVT or endovascular treatment at our centre between 2010 and 2012 was analyzed. Parameters included age, National Institutes of Health Stroke Scale (NIHSS) on admission, door to needle time, stroke subtype, modality of treatment, outcome based on modified Rankin Scale (mRS) Score at 90 days follow up and mortality rates at 90 days. Statistical Analysis: Tabulated results were analysed using INSTAT Graphpad analyser. Data were analysed using paired and unpaired $t$-test, Chi-square test, and Fishers test as applicable. $P$ value was considered significant when it was $<0.05$. Results: Upon comparison of the outcomes of patients with acute ischemic stroke and large vessel disease treated with endovascular therapy with those treated with IVT, it was found that the former group had better outcomes. We also found that in spite of there being a significant difference in the NIHSS on admission and a significant difference in the door to needle time, the outcomes of patients treated using intravenous or endovascular therapy were similar. There was no statistically significant difference in mortality rates between intravenous and endovascular groups. Conclusions: IVT is currently the standard of care in the management of acute ischemic stroke. Endovascular treatment during the window period is reserved for those patients with contraindication to IVT. In this study, we found that patients with documented large vessel disease with no evidence of cross flow through Willisian collaterals benefit from endovascular treatment. We recommend that all patients of acute ischemic stroke, be subjected to a baseline angiogram either computed tomography $(\mathrm{CT})$ or magnetic resonance imaging $(\mathrm{MRI})$ to document vessel status. This will help in identifying patients who may benefit from early endovascular treatment, if they fail to improve with IVT. Further, large trials using stent retrievers are needed, to prove that endovascular treatment is superior to IVT, in presence of documented large vessel disease.
\end{abstract}

Key words: Endovascular thrombolysis, intravenous thrombolysis, IV thrombolysis, stent retriever, stroke

\begin{tabular}{|l|l|}
\hline \multicolumn{2}{|c|}{ Access this article online } \\
\hline Quick Response Code: & Website: \\
\hline & www.ruralneuropractice.com \\
\cline { 2 - 2 } & \\
\hline & \\
\hline
\end{tabular}

\section{Introduction}

The management of acute ischemic stroke has undergone a sea of change with the introduction of intravenous thrombolysis (IVT). In the NINDS ${ }^{[1]}$ trial, a clear benefit was found in patients who were treated with IVT within the first 3 hours. Following the results of the ECASS $3^{[2]}$ trial, the window period for IVT was extended to 4.5 hours.

\section{Address for correspondence:}

Dr. Vikram Huded, Department of Neurology, Narayana Hrudayalaya Institute of Neurosciences, Hosur Road, Bangalore, Karnataka, India. E-mail: drvikramhuded@gmail.com 
As per the PROACT ${ }^{[3]}$ trial, patients of acute ischemic stroke with a middle cerebral artery occlusion may be treated using intraarterial thrombolysis within $6 \mathrm{~h}$. The MERCI, ${ }^{[4]}$ Multi Merci, ${ }^{[5]}$ and Penumbra ${ }^{[6]}$ trials showed better recanalisation in patients having large vessel occlusion who were treated with endovascular therapy. Recently published data from three large trials, IMS 3, ${ }^{[7]}$ Synthesis Expanision, ${ }^{[8]}$ and MR rescue ${ }^{[9]}$ failed to demonstarte the superiority of endovascular treatment over IVT. In these trials, stent retrievers were used in very few patients. However, according to SWIFT ${ }^{[10]}$ trial stent retrievers have better recanalisation rates and therefore, better outcomes. We present our results, from a tertiary care center in Bangalore, comparing the outcomes in patients of acute ischemic stroke treated with IVT versus those treated using endovascular therapy. The endovascular treatment was done by a interventional neurologist and in a majority of cases stent retrievers were used.

The stroke protocol followed in our institute is described in Figure 1. All patients with acute stroke undergo a baseline imaging, comprising of either a computed tomography (CT) with CT angiogram or a magnetic resonance imaging (MRI) imaging with stroke protocol. This comprises of a diffusion sequence, an ADC, T2, FLAIR, and a TOF angio of neck and intracranial vessels. As the MRI is more time consuming, it was reserved for patients who presented with a wake up stroke or patients with posterior circulation stroke who presented beyond the window period. Thus in all patients with acute ischemic stroke, an angiogram of neck and intracranial vessels was available before starting thrombolysis.

If the angiogram was normal, patients were given IVT. If the angiogram showed a large vessel occlusion along with either contraindications for IVT or no improvement with IVT, then they were taken for endovascular therapy. In some cases, before the IMS3 results, few patients were taken directly for endovascular treatment after taking informed consent from the patients relatives.

We compared the results of patients who underwent IVT with those of patients who underwent endovascular therapy. This study also compared the outcomes of patients with large vessel occlusion who were treated with endovascular therapy with those who received IVT.

\section{Materials and Methods}

Patients with acute ischemic stroke presenting to our institute who underwent thrombolysis were included in this study. In total, 53 patients were thrombolysed between 2010 and 2012; 23 received IVT and 30 were taken up for endovascular treatment. We present an analysis of their outcome.

Patients were divided into two groups depending on whether they received intavenous thrombolysis or endovascular treatment. They were analysed based on their National Institutes of Health Stroke Scale (NIHSS) on admission, time from onset of symptoms, door to needle time, stroke subtype, modified Rankin Scale (mRS), and mortality at 90 days.

The outcome of the patients who received IVT was analysed based on angiogram results showing normal study or on an evidence of a large vessel occlusion.

We also compared the outcomes of patients who received IVT, in presence of documented large vessel disease to the outcomes of patients who underwent endovascular treatment.

Of the 30 patients of the endovascular group, 23 patients underwent endovascular therapy within $4.5 \mathrm{~h}$ from the onset of symptoms, that is, the window period for IVT. The outcome in these patients was compared with those patients who received IVT in the presence of a large vessel occlusion.

Tabulated results were analysed using INSTAT Graphpad analyser. Data were analysed using paired and unpaired $t$-test, Chi-square test, and Fishers test as applicable. $P$ value was considered significant when it was less than 0.05 .

\section{Results}

A total of 53 patients underwent thrombolysis between 2010 and 2012; 23 patients underwent IVT and 30 patients underwent endovascular treatment [Table 1].

The patients who received IVT were subdivided on the basis of their CT/MR angiograms into those having a normal angiogram and those with evidence of a large vessel occlusion. Out of the 23 patients, 14 had a normal baseline angiogram. Nine patients had documented large vessel disease. Among the nine patients with documented large vessel, four patients had cross flow on their angiogram (CT or MR angiogram) [Table 2].

Further, we compared the patients who underwent IVT in the presence of documented large vessel disease with the patients of the endovascular group [Table 3]. 
Stroke alert activated by Emergency Room Physician<smiles>C1CCCC1</smiles>

\section{Duty Neurologist, Duty Radiologist, Duty Anaesthetist,} Radiology Technician, Cath Lab Technician, Informed

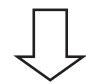

NIHS Assessed, Bloods ent for CBC/KFT/LFT/PT, INR/ECG done

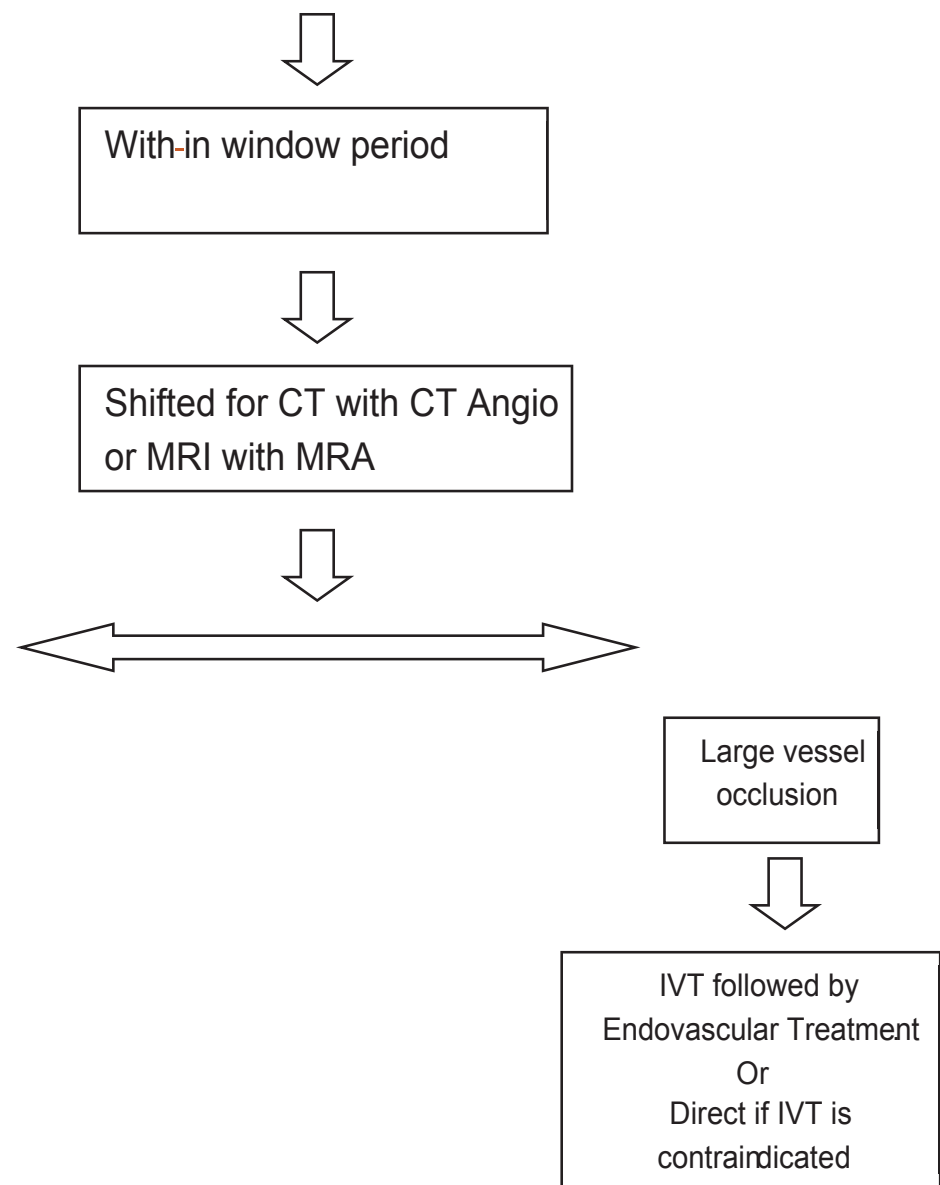

Figure 1: Stroke Protocol followed at our institute

Outcomes of patients who received endovascular treatment within $4.5 \mathrm{~h}$ and of the intravenous group patients with documented large vessel disease were compared [Table 4].

\section{Discussion}

A total of 53 patients with acute ischemic stroke underwent thrombolysis between 2010 and 2012; 30 patients underwent endovascular treatment and 23 underwent IVT. Nine of the patients who underwent IVT had a large vessel occlusion. Patients with occlusion of one internal carotid artery were considered as having good cross flow if the MCA was filled through anterior communicating artery (ACOM) or posterior communicating artery (PCOM) (Willisian Collaterals) [Figures 2 and 3]. Four of nine patients had a documented cross flow. 
Table 1: Comparision of intravenous group and intervention group

\begin{tabular}{lccc}
\hline & $\begin{array}{c}\text { Intravenous } \\
\text { group }\end{array}$ & $\begin{array}{c}\text { Intervention } \\
\text { group }\end{array}$ & $\boldsymbol{P}$ value \\
\hline Mean age & 49.21 & 49.53 & $>0.05$ \\
Mean time (onset to door) & $137 \mathrm{~min}$ & $218 \mathrm{~min}$ & 0.0175 \\
Door to needle time & $66.25 \mathrm{~min}$ & $117.50 \mathrm{~min}$ & $<0.0001$ \\
Type of stroke & & & \\
Large vessel & 7 & 13 & \\
Cardio embolic & 5 & 16 & \\
Stroke of other determined & 1 & 1 & \\
cause & & & \\
Stroke of undetermined & 10 & & \\
cause/lacunar & & & \\
Mean NIHSS & 14.60 & 20.4 & $<0.05$ \\
Median NIHSS & 14 & 20 & $<0.05$ \\
Median MRS at FU & 2 & .5 & $>0.05$ \\
MRS<2 at FU & $14(60.8 \%)$ & $19(63.3 \%)$ & $>0.005$ \\
MRS 0 at FU & $8(34.7 \%)$ & $15(50 \%)$ & $>0.005$ \\
90 days mortality & $5(21.7 \%)$ & $5(16.6 \%)$ & $>0.005$ \\
Symptomatic ICH & 2 & 0 & \\
\hline
\end{tabular}

NIHSS: National institutes of health stroke scale, MRS: Modified rankin scale, FU: Follow up, ICH: Intracranial hemorrhage

Table 3: Comparision of patients with large vessel occlusion who underwent IVT with endovascular treatment

\begin{tabular}{lcc}
\hline & $\begin{array}{c}\text { IVT with large } \\
\text { vessel occlusion }\end{array}$ & $\begin{array}{c}\text { Endovascular } \\
\text { group }\end{array}$ \\
\hline Total no & 9 & 30 \\
Mean age & 47.9 & 49.53 \\
Mean time & $142 \mathrm{~min}$ & $218 \mathrm{~min}$ \\
Median NIHSS & 20 & 20 \\
Territory/vessels & MCA-5* & MCA $16(53 \%)$ \\
& ICA-4* & ICA-10 (33\%) \\
TIMI>2 & NA & BA-4 (13\%) \\
Median MRS & 3 & $23(76 \%)$ \\
MRS<2 & $4(44 \%)^{* *}$ & 0.5 \\
90 days mortality & $3(33.3 \%)$ & $19(63 \%)$ \\
\hline
\end{tabular}

NIHSS: National institutes of health stroke scale, MRS: Modified rankin scale, IVT: Intravenous thrombolysis, NA: Not applicable, ICH: Intracranial hemorrhage, ICA: Internal carotid artery, TIMI: Thrombolysis in myocardial infarction, MCA: Middle cerebral artery, BA: Basilar artery. ${ }^{\star} \mathrm{ICA}$ origin to M1 occluded
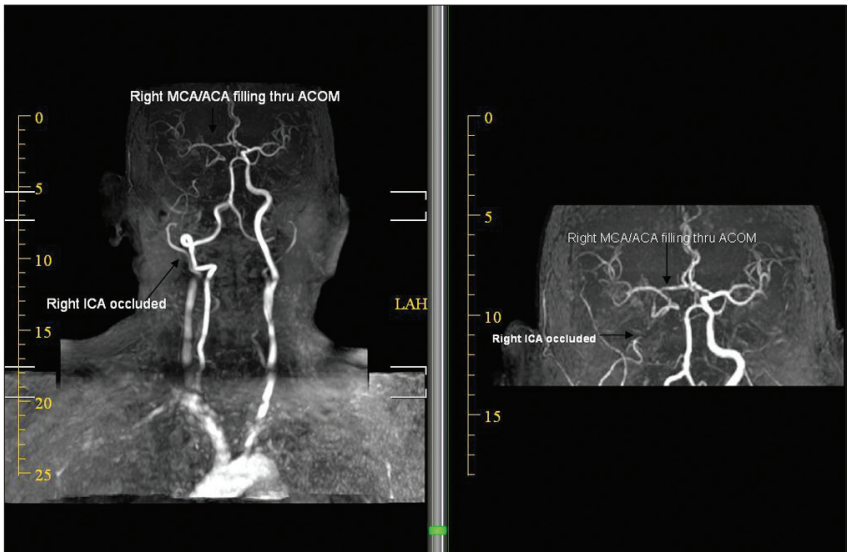

Figure 2: TOF MR angiogram of a patient demonstrating cross flow. The Right ICA is occluded, right MCA and ACA filling through ACOM
In the earlier part of our study, stent retrievers were used only if there was no recanalization after intraarterial injection of $20 \mathrm{mg}$ of rTPA (Recombinant tissue plasminogen activator). Toward the later part of our study, stent retrievers (Solitaire) were used as primary modality of endovascular treatment. Two patients who underwent endovascular treatment were initially given intravenous rTPA (bridging thrombolysis). Stent retrievers (Solitaire) were used in 21 out of 30 patients during the study. The nine patients who underwent IVT in the presence of large vessel occlusion were not

Table 2: Comparision of patients who underwent IVT with or without large vessel occlusion

\begin{tabular}{lcc}
\hline & $\begin{array}{c}\text { IVT with large } \\
\text { vessel occlusion }\end{array}$ & $\begin{array}{c}\text { IVT with normal } \\
\text { angiogram }\end{array}$ \\
\hline Total no & 9 & 14 \\
Mean age & 47.9 & 50.1 \\
Mean time & $142 \mathrm{~min}$ & $134 \mathrm{~min}$ \\
Median NIHSS & 20 & 11 \\
Territory/vessels & $P<0.001$ & \\
& MCA-5* & MCA 13 \\
Median MRS & ICA-4* & BA-1 \\
MRS 2 & 3 & 1 \\
90 days mortality & $4(44 \%)^{* *}$ & $10(71 \%)$ \\
\hline
\end{tabular}

IVT: Intravenous thrombolysis, MRS: Modified rankin scale, NIHSS: National institutes of health stroke scale, ICA:Internal carotid artery, MCA: Middle cerebral artery, BA: Basilar artery. ${ }^{\star} \mathrm{ICA}$ origin to M1 occluded

Table 4: Comparison of patients with large vessel occlusion within $4.5 \mathrm{hrs}$ of stroke onset

\begin{tabular}{lccc}
\hline & $\begin{array}{c}\text { Large vessel } \\
\text { IVT } \mathbf{N = 9}\end{array}$ & $\begin{array}{c}\text { Endovascular } \\
\text { group } \boldsymbol{N = 2 3}\end{array}$ & $\boldsymbol{P}$ value \\
\hline Age & 47.88 & 46.72 & 0.848 \\
Time from onset & 2.22 & 2.36 & 0.793 \\
NIHSS & 20 & 18 & 0.479 \\
Median MRS at 90 days & 3 & 0.5 & $<0.05$ \\
MRS $<2$ at 90 days & $4^{* *}(44.44 \%)$ & $19(82.60 \%)$ & 0.0745 \\
MRS $<2$ at 90 days with & 0 & $19(82.60 \%)$ & $<0.0013$ \\
no cross flow & & &
\end{tabular}

NIHSS: National institutes of health stroke scale, MRS: Modified rankin scale, IVT: Intravenous thrombolysis. ${ }^{* *}$ All 4 patients had documented cross flow

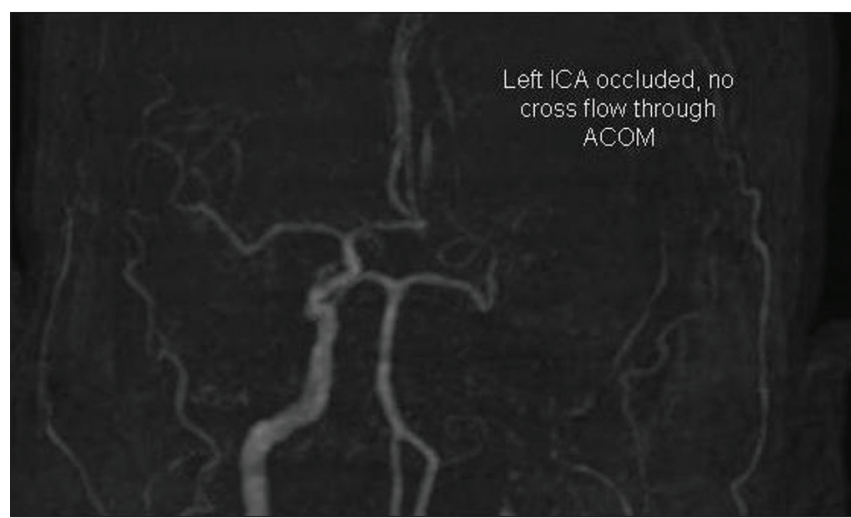

Figure 3: TOF MR angiogram showing ICA occluded with no evidence of cross flow 
taken for endovascular therapy, primarily because the relatives did not give consent. The main constraint was financial.

A statistically significant difference was found between the median NIHSS on admission in the endovascular group (median NIHSS 20) and the intravenous group (median NIHSS 14). The 'door to needle' time in the endovascular group was $117.50 \mathrm{~min}$ versus $66.25 \mathrm{~min}$ in the intravenous group. This difference was also statistically significant. Despite the significant difference in the NIHSS on admission and in 'door to needle' time, there were no statistically significant differences between the two groups in the median mRS at 90 days follow up ( 3 in the intravenous group and 0.5 in the endovascular group) and in the 90 days mortality rate. Two patients in the study had a symptomatic intracerebral hemorrhage; both were in the intravenous group.

In the intravenous group, the NIHSS on admission was statistically higher in the patients who had a large vessel occlusion (median NIHSS 20) as compared with those who had a normal angiogram (median NIHSS 11), suggesting that the patients with large vessel occlusion had a more severe stroke. On comparing the outcomes between these two subgroups, the median mRS achieved by the nine patients with large vessel occlusion was 3 as compared with a median mRS of 1 achieved by patients with a normal angiogram. This difference was not statistically significant. Three of the 9 patients (33\%) with large vessel occlusion and 2 of the 14 patients (14\%) with a normal angiogram were dead at 90 days follow-up. These findings are summarized in Table 2.

Comparing the endovascular group with the patients who received IVT in the presence of large vessel occlusion, the median NIHSS was 20 in both groups. The median mRS at 90 days was 0.5 in the endovascular group and 3 in the intravenous group. A total of $63 \%$ of the endovascular group achieved a mRS of $<2$ as compared with the intravenous group in which $44 \%$ achieved a mRS of $<2$ at 90 days follow up. The mortality in the endovascular group was $16 \%$ (5 out of 30 ) as compared with $33 \%$ (3 out of 9) in the intravenous group with large vessel occlusion. There was no statistical difference in the outcomes between the two groups.

Of the 30 patients, 23 in the endovascular group were treated within $4.5 \mathrm{~h}$, that is, the window period for IVT. Of these 23 patients, 2 received IVT prior to being taken for endovascular treatment. The remaining received endovascular treatment as many had contraindications for IVT, while a few were taken directly for endovascular therapy, before the IMS3 results were published. We compared this subset of patients with the patients who received IVT in the presence of large vessel occlusion. The therapy in these patients was also within $4.5 \mathrm{~h}$. The median mRS in the endovascular group was 0.5 as compared with the intravenous group, which was 3. This difference was found to be statistically significant. A total of $82 \%$ (19 out of 23 patients) had a mRS of 2 or less at 90 days in the endovascular group as compared with $44 \%$ (4 out of 9 ) in the intravenous group. In four out of nine patients in the intravenous group with large vessel occlusion, there was no cross flow through Willisian collaterals. None of these patients achieved a mRS of $<2$ at 90 days follow up as compared with the 19 out of 23 patients in the endovascular group. This difference was found to be statistically significant.

In the IMS3 ${ }^{[7]}$ trial, patients were randomly assigned to receive intervention following intravenous TPA and compared with patients who received intravenous TPA alone. They concluded that there was no improvement in functional independence in patients who had received only intravenous TPA or patients who had received intravenous TPA as well as intervention treatment. In the Synthesis Trial, ${ }^{[8]}$ patients were randomly assigned to be treated by IVT or intervention. The intervention modality used was intraarterial thrombolysis, mechanical, or a combination of both. It was concluded that there was no difference between the two groups. In both of these trials, vessel status was not documented in all patients. Stent retrievers were used only in a few patients.

In this study, patients who received endovascular therapy fared just as well as those who received intravenous therapy in spite of having a significantly higher NIHSS, longer duration of stroke, and a higher 'door to needle' time. The mortality rates remained similar between the two groups.

Patients who underwent IVT with documented large vessel disease, with no cross flow on angiogram fared poorly as compared with patients treated with endovascular modalities.

In patients treated within $4.5 \mathrm{~h}$ of symptom onset, the endovascular group had a better outcome compared with intravenous group with documented large vessel disease.

It is recommended that all patients presenting with acute ischemic stroke in the window period should have baseline angiogram either CT with angiogram or MRI with angiogram.

Patients with no large vessel occlusion should undergo IVT. 
However, patients who have documented M1, carotid T, or basilar occlusion should be considered for endovascular treatment as early as possible if they fail IVT or have contraindications for IVT.

The limitation of this study was that it was an observational nonrandomized study with a small sample size.

Further studies with larger statistical sample size are needed to confirm the added benefit of endovascular treatment using stent retrievers in patients with documented large vessel disease. Demonstration of large vessel occlusion along with NIHSS should be considered criteria for randomization.

\section{References}

1. Tissue plasminogen activator for acute ischemic stroke. The National Institute of Neurological Disorders and Stroke rt-PA Stroke Study Group. N Engl J Med 1995;333:1581-7.

2. Hacke W, Kaste M, Bluhmki E, Brozman M, Dávalos A, Guidetti D, et al. ECASS Investigators. Thrombolysis with alteplase 3 to 4.5 hours after acute ischemic stroke. N Engl J Med 2008;359:1317-29.

3. del Zoppo GJ, Higashida RT, Furlan AJ, Pessin MS, Rowley HA, Gent M. PROACT: A phase II randomized trial of recombinant pro-urokinase by direct arterial delivery in acute middle cerebral artery stroke. PROACT Investigators. Prolyse in acute cerebral thromboembolism. Stroke 1998;29:4-11.
4. Smith WS, Sung G, Starkman S, Saver JL, Kidwell CS, Gobin YP, et al. MERCI Trial Investigators. Safety and efficacy of mechanical embolectomy in acute ischemic stroke: Results of the MERCI trial. Stroke 2005;36:1432-8.

5. Smith WS, Sung G, Saver J, Budzik R, Duckwiler G, Liebeskind DS, et al. Multi MERCI Investigators. Mechanical thrombectomy for acute ischemic stroke: Final results of the multi MERCI trial. Stroke 2008;39:1205-12.

6. Penumbra Pivotal Stroke Trial Investigators. The penumbra pivotal stroke trial: Safety and effectiveness of a new generation of mechanical devices for clot removal in intracranial large vessel occlusive disease. Stroke 2009;40:2761-8

7. Broderick JP, Palesch YY, Demchuk AM, Yeatts SD, Khatri P, Hill MD, et al. Interventional Management of Stroke (IMS) III Investigators. Endovascular therapy after intravenous t-PA versus t-PA alone for stroke. N Engl J Med 2013;368:893-903.

8. Ciccone A, Valvassori L, Nichelatti M, Sgoifo A, Ponzio M, Sterzi R, et al. SYNTHESIS Expansion Investigators. Endovascular treatment for acute ischemic stroke. N Engl J Med 2013;368:904-13.

9. Kidwell CS, Jahan R, Gornbein J, Alger JR, Nenov V, Ajani Z, et al. MR RESCUE Investigators. A trial of imaging selection and endovascular treatment for ischemic stroke. N Engl J Med 2013;368:914-23.

10. Saver JL, Jahan R, Levy EI, Jovin TG, Baxter B, Nogueira R, et al. The SWIFT Trialists SOLITAIRE ${ }^{\mathrm{TM}}$ with the intention for thrombectomy (SWIFT) trial: Design of a randomized, controlled, multicenter study comparing the SOLITAIRETM flow restoration device and the MERCI retriever in acute ischaemic stroke. Int J Stroke 2012 [Epub ahead of print].

How to cite this article: Huded V, De Souza R, Nagarajaiah RK, Zafer SM, Nair R, Acharya H. Thrombolysis in acute ischemic stroke: Experience from a tertiary care centre in India. J Neurosci Rural Pract 2014;5:25-30.

Source of Support: Nil. Conflict of Interest: None declared. 